\title{
Fabrication of Functionalized MOFs Incorporated Mixed Matrix Hollow Fiber Membrane for Gas Separation
}

\author{
Haitao Zhu, ${ }^{1,2}$ Xingming Jie, ${ }^{1}$ and Yiming Cao ${ }^{1}$ \\ ${ }^{1}$ Dalian Nation Library for Clean Energy (DNL), Dalian Institute of Chemical Physics, Chinese Academy of Sciences, \\ Dalian 116023, China \\ ${ }^{2}$ University of Chinese Academy of Sciences, Beijing 100049, China
}

Correspondence should be addressed to Xingming Jie; jiexm@dicp.ac.cn and Yiming Cao; ymcao@dicp.ac.cn

Received 4 November 2016; Accepted 6 February 2017; Published 26 February 2017

Academic Editor: Hossein Kazemian

Copyright (C) 2017 Haitao Zhu et al. This is an open access article distributed under the Creative Commons Attribution License, which permits unrestricted use, distribution, and reproduction in any medium, provided the original work is properly cited.

\begin{abstract}
The metal-organic framework (MOFs) of MIL-53 was functionalized by aminosilane grafting and then incorporated into Ultem ${ }^{\circledR} 1000$ polymer matrix to fabricate mixed matrix hollow fiber membrane (MMHFM) with high separation performance. SEM, XRD, and TGA were performed to characterize the functionalized MIL-53 and prepared MMHFM. The filler particles were embedded in membrane successfully and dispersed well in the polymer matrix. The incorporation of MOFs endowed MMHFM better thermal stability. Moreover, effects of solvent ratio in spinning dope, spinning condition, and testing temperature on gas separation performance of MMHFM were investigated. By optimizing dope composition, air gap distance, and bore fluid composition, MMHFM containing functionalized MIL-53 achieved excellent gas permeance and $\mathrm{CO}_{2} / \mathrm{N}_{2}$ selectivity. The CO permeance increased from $12.2 \mathrm{GPU}$ for pure Ultem HFM to $30.9 \mathrm{GPU}$ and the ideal $\mathrm{CO}_{2} / \mathrm{N}_{2}$ selectivity was enhanced from 25.4 to 34.7 simultaneously. Additionally, gas permeance increased but the selectivity decreased with the temperature increase, which followed the solution-diffusion based transport mechanism.
\end{abstract}

\section{Introduction}

Membrane based gas separation and purification hold promise as energy-efficient technology if compared with traditional methods such as adsorption and cryogenic distillation [1-4]. However, the permeability-selectivity tradeoff described as Robeson upper bound restricted gas separation performance of polymeric membranes [5]. Several solutions have been proposed in past decades to further improve membrane performance. One of the most effective routes to enhance the separation performance is fabrication of mixed matrix membrane (MMM) that combines inorganic materials with polymer matrix $[6,7]$. Regarding the filler materials, metal-organic frameworks (MOFs) are a satisfactory alternative to improve the permeation properties of membranes because of its large surface area, high porosity, adsorption capacity, and good compatibility [8-10].

Over the past few years, various MOFs have been incorporated into membranes to improve gas separation performance, such as Matrimid ${ }^{\circledR 5218 / Z I F-8 ~[11], ~ P B I / Z I F-7 ~}$

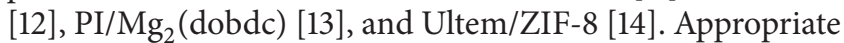
structures of MOFs are efficient for gas separation application as previous references reported [15-19]. Couck et al. [20] revealed that the amino-functionalized $\mathrm{MOFs} \mathrm{NH}_{2}$-MIL-53 achieved excellent performance for $\mathrm{CO}_{2}$ capture. Moreover, MMM based on $\mathrm{NH}_{2}$-MIL-53 with improved structural feature and gas separation performance was synthesized by incorporating the filler particles in polyimide matrix. The membrane containing $25 \mathrm{wt} \%$ MOFs loading presented a $50 \%$ increment in $\mathrm{CO}_{2}$ permeability, while retaining the separation selectivity [21]. The amino-functionalized MOFs filled MMMs have been also fabricated by Seoane et al. and the membrane performance was improved approaching the Robeson $\mathrm{CO}_{2} / \mathrm{CH}_{4}$ and $\mathrm{H}_{2} / \mathrm{CH}_{4}$ upper bound with $10 \mathrm{wt} \% \mathrm{MOF}$ loading [22]. In addition, other researchers have fabricated mixed matrix composite membrane by synthesizing MOFs continuous membrane on outer-side or inner-side of hollow fiber membrane to improve gas separation performance [23-26]. 
However, the filler particle aggregation and interfacial isolation are the major drawbacks that hinder the development of inorganic filler based MMMs [27, 28]. As a feasible method, the certain functions on MOFs could lead to enhanced interaction with functional groups of polymer chains [28]. For example, in NH2-MIL-53 incorporated MMM, the hydrogen bonding between the sulfone group of polysulfone and amine of MOFs enhanced compatibility at the polymer/filler interface [29]. Here, the present work prepared functionalized MOFs by grafting aminosilane to improve the interfacial affinity between filler particles and polymer matrix. The $-\mathrm{NH}_{2}$ group on the functionalized MOFs could interact with carbonyl group of Ultem 1000 and the grafting alkyl chains also enhanced the adhesion with polymer matrix. Moreover, the dispersion of filler particles was uniform in the aid of silicane modification. In order to prepare asymmetric MMHFM with optimal membrane structure and remarkable separation performance, we investigated the important factors including solvent/nonsolvent ratio in spinning dope solution, spinning condition (air gap distance and bore fluid composition), and testing temperature.

\section{Experimental}

2.1. Materials. Ultem 1000 polymer was purchased from GE Plastics in pellet form and was dried $140^{\circ} \mathrm{C}$ for $12 \mathrm{~h}$ before use. Terephthalic acid $\left(\mathrm{H}_{2} \mathrm{BDC}\right)$ was purchased from Sinopharm Chemical Reagent Co., Ltd. 3-Aminopropyltrimethoxysilane (APTMS) was purchased from J\&K Scientific Ltd., Beijing, China. N,N-dimethylformamide (DMF), $\mathrm{Al}\left(\mathrm{NO}_{3}\right)_{3} \cdot 9 \mathrm{H}_{2} \mathrm{O}$, $\mathrm{N}$-methyl-2-pyrrolidone (NMP), anhydrous ethanol (EtOH), n-hexane, petroleum ether, and aqueous ammonia (25 wt\%) were purchased from Kemiou Chemical Reagent Co., Ltd., Tianjin, China. Polydimethylsiloxane (PDMS, Sylgard 184) was produced by Dow Chemicals.

2.2. Synthesis of MIL-53. MIL-53 was synthesized by the solvothermal method [30, 31]. First, $\mathrm{Al}\left(\mathrm{NO}_{3}\right)_{3} \cdot 9 \mathrm{H}_{2} \mathrm{O}(4.22 \mathrm{~g})$ and $\mathrm{H}_{2} \mathrm{BDC}(1.245 \mathrm{~g})$ were added into the mixed solvent of $20 \mathrm{~mL}$ of deionized water and $55 \mathrm{~mL}$ of DMF and magnetically stirred for $40 \mathrm{~min}$. Then the solution was transferred into a Teflon-lined steel autoclave and heated in an air oven at $150^{\circ} \mathrm{C}$ for $48 \mathrm{~h}$. The product was centrifuged and washed with deionized water three times, followed by immersing in DMF for $8 \mathrm{~h}$. Afterward, the white powder was filtered from $\mathrm{DMF}$ and dried in an air oven for $12 \mathrm{~h}$ at $150^{\circ} \mathrm{C}$. Finally, the fine powdered MIL-53 was further activated at $180^{\circ} \mathrm{C}$ under vacuum for $12 \mathrm{~h}$.

2.3. Functionalization of MIL-53. MIL-53 particles ( $2.0 \mathrm{~g})$ were dispersed in the mixed solvent of $2.6 \mathrm{~g}$ of deionized water, $60 \mathrm{~mL}$ of $\mathrm{EtOH}$, and $1.36 \mathrm{~g}$ of aqueous ammonia. The suspension was stirred and sonicated in a round-bottomed flask at $60^{\circ} \mathrm{C}$ for $2 \mathrm{~h}$. Then $1.44 \mathrm{~g}$ of APTMS was added dropwise into the mixture under stirring. The suspension reacted at room temperature for $12 \mathrm{~h}$ and then was centrifuged to obtain the modified product and washed with
$\mathrm{EtOH}$ at least three times to remove residual APTMS. Finally, the aminosilane modified MIL-53 was dried under vacuum at $80^{\circ} \mathrm{C}$ for $24 \mathrm{~h}$.

\subsection{Mixed Matrix Hollow Fiber Membrane Preparation}

2.4.1. Preparation of Spinning Dope. A certain amount of functionalized MIL-53 was added to a portion of the required NMP with stirred and sonicated alternatively for $45 \mathrm{~min}$ to obtain a well-dispersed suspension. Then MOFs filler was primed by addition of a dilute polymer solution into the suspension to ensure better adhesion. This mixture was stirred for $3 \mathrm{~h}$ and sonicated for $45 \mathrm{~min}$ until it displayed in uniform consistency. Then a required amount of EtOH was added with the remaining NMP into the suspension. Finally, the Ultem 1000 pellet was gradually added into the mixture to increase dope viscosity. After adding all the components, the suspension was mechanically stirred at $60^{\circ} \mathrm{C}$ for about $12 \mathrm{~h}$ to fully dissolve the polymer. For comparison purposes, the pure Ultem dope solution was prepared following an identical procedure. The polymer concentration in all dope solutions kept 33 wt $\%$ and the MOFs loading kept 15 wt $\%$ which was determined by

$$
\begin{aligned}
\text { MOFs loading }(w t \%)= & \left(\frac{\text { wt. MOFs }}{\text { wt. MOFs }+ \text { wt. polymer }}\right) \\
& \times 100 \% .
\end{aligned}
$$

2.4.2. MMHFM Fabrication. Asymmetric hollow fiber membranes in this study were fabricated via the dry-wet spinning method. The homogeneous dope and bore fluid were coextruded from a spinneret after dope solution was degassed; then the nascent fiber entered a water coagulant bath with a range of spinning parameters (Table 1) through the air gap distance and was wound up on a take-up drum. The spinning process was performed at $60^{\circ} \mathrm{C}$ and a filter was attached upstream of the spinneret to prevent impurity particles blocking the narrow spinneret orifice. Proper pressure was supplied by the nitrogen cylinder to maintain a constant spinning rate and a syringe pump was used to control the flow rate of bore fluid.

The as-fabricated fibers were rinsed in flowing tap water for about $24 \mathrm{~h}$ to remove the remaining solvent. Then the fibers were exchanged by immersing in three successive aliquots of $\mathrm{EtOH}$ for $30 \mathrm{~min}$, followed by $30 \mathrm{~min}$ immersion in three successive aliquots of n-hexane. Afterward, the hollow fiber membranes were dried at $70^{\circ} \mathrm{C}$ for $3 \mathrm{~h}$.

For sealing the microvoids or defects in the skin layer, each membrane module containing fibers was dip-coated by $3 \mathrm{wt} \%$ Sylgard 184 solution with a corresponding curing agent (10 wt $\%$ of Sylgard 184 PDMS) in petroleum ether. After coating for $30 \mathrm{~min}$, the modules were placed into a $70^{\circ} \mathrm{C}$ oven and cured for $3 \mathrm{~h}$ after petroleum ether evaporation. The coated hollow fiber membranes were tested to evaluate gas permeation properties.

2.5. Characterizations. The morphologies of functionalized MOFs and membranes were examined by scanning electron 
TABLE 1: Detailed spinning parameters of hollow fiber membrane fabrication.

\begin{tabular}{lc}
\hline Spinning parameters & Value \\
\hline Spinneret dimension $(\mathrm{mm})$ & o.d./i.d. $=0.70: 0.35$ \\
Spinneret temperature $\left({ }^{\circ} \mathrm{C}\right)$ & $60 \pm 2$ \\
Air gap distance $(\mathrm{cm})$ & $0.8-5.0$ \\
& $\mathrm{NMP}: \mathrm{H}_{2} \mathrm{O}=10 \mathrm{wt} \%: 90 \mathrm{wt} \%$ \\
Bore fluid composition & $\mathrm{NMP}: \mathrm{H}_{2} \mathrm{O}=50 \mathrm{wt} \%: 50 \mathrm{wt} \%$ \\
& $\mathrm{NMP}: \mathrm{H}_{2} \mathrm{O}=90 \mathrm{wt} \%: 10 \mathrm{wt} \%$ \\
Bore fluid flow rate $(\mathrm{mL} / \mathrm{min})$ & $0.27 \pm 0.02$ \\
External coagulant & Tap water \\
Temperature of external coagulant $\left({ }^{\circ} \mathrm{C}\right)$ & $18 \pm 2$ \\
\hline
\end{tabular}

microscopy (SEM, Zeiss Supra 55 Sapphire). MOFs particles were tested without any dispersion. The membrane samples were frozen and broken off in liquid nitrogen and then sputtered with gold before SEM testing. MOFs and hollow fiber membranes were characterized by powder X-ray diffraction (XRD) using a PANalytical Empyrean diffractometer with $\mathrm{Cu} \mathrm{K} \alpha$ radiation $(\lambda=0.15406 \mathrm{~nm})$. The basal spacing was calculated by the Bragg's equation:

$$
n \lambda=2 d \sin \theta .
$$

Thermal stability of functionalized MIL-53, pure Ultem HFM, and MMHFM were measured by thermo-gravimetric analysis (TGA) at a heating rate of $10^{\circ} \mathrm{C} / \mathrm{min}$ from room temperature to $800^{\circ} \mathrm{C}$ under nitrogen atmosphere using a TGA instrument (Netzsch STA449F3).

2.6. Pure Gas Permeation Measurement. Gas permeation experiments were conducted at $35^{\circ} \mathrm{C}$ with a testing pressure of $0.5 \mathrm{MPa}$. The permeation flow rate was measured with soap bubble flow meter and permeance $(P / l)$ was calculated by the following:

$$
\frac{P}{l}=\frac{p_{p} V_{P}}{R T} \frac{V_{m}}{A t \Delta p},
$$

where $p_{p}$ is the pressure of the permeate side (cmHg), $V_{P}$ is the volume of permeate gas during the test duration $\left(\mathrm{cm}^{3}\right)$, $V_{m}$ is the molar volume of ideal gas at standard condition $\left[\mathrm{cm}^{3}\right.$ (STP)/mol], $R$ is the universal gas constant, $T$ is the absolute temperature $(\mathrm{K}), A$ is the permeation area $\left(\mathrm{cm}^{2}\right)$, $t$ is the test duration (s), and $\Delta p$ is the trans-membrane pressure difference $(\mathrm{cmHg})$. The permeance $(P / l)$ values are commonly reported in the gas permeation unit GPU, $1 \mathrm{GPU}$ $=1 \times 10^{-6} \mathrm{~cm}^{3}(\mathrm{STP}) /\left(\mathrm{cm}^{2} \cdot \mathrm{s} \cdot \mathrm{cmHg}\right)$. The ideal selectivity of gases $i$ and $j$ is defined as follows:

$$
\alpha_{(i / j)}=\frac{(P / l)_{i}}{(P / l)_{j}} .
$$

For each sample, at least three membrane modules were prepared and tested as our previous work [3], and the average value of testing result (with standard deviation) was used to determine the gas separation performance.

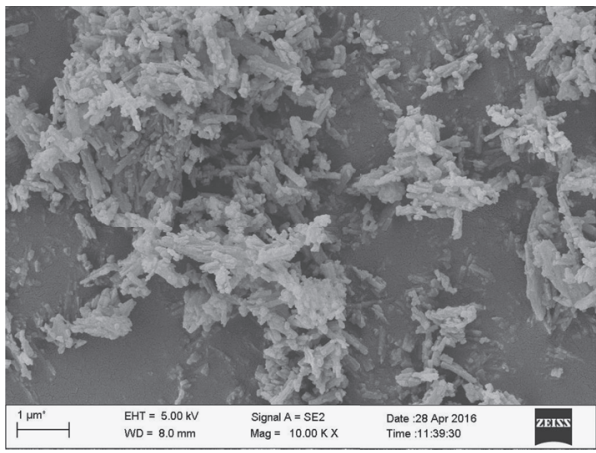

FIGURE 1: SEM image of functionalized MIL-53 powder.

\section{Results and Discussion}

3.1. Characterizations. Figure 1 depicts SEM image of the functionalized MIL-53 by grafting aminosilane and MOFs particles appeared in the clump form due to the particle agglomeration. Therefore, the functionalized MOFs used as filler in MMHFM should be sufficiently dispersed before adding into spinning dope solution. The outer and inner surface, cross section, and skin layer of prepared pure Ultem HFM were shown in Figure 2. Morphology of MMHFM with $15 \mathrm{wt} \%$ filler loading was presented in Figure 3. The distribution of MOFs particles in Ultem matrix was homogeneous and there was no obvious aggregation as indicated by EDX mapping. Moreover, no surface cracks or interfacial voids revealed the excellent compatibility between the MOFs filler and polymer matrix. Additionally, more finger-like pores were generated in the outer region of MMHFM, which should be caused by instantaneous demixing with addition of MOFs particles.

The X-ray diffraction (XRD) patterns of functionalized MIL-53 and hollow fiber membranes are shown in Figure 4. The Ultem/functionalized MIL-53 MMHFM exhibited new peaks compared with pure Ultem HFM, which indicated the presence of functionalized MIL-53 particles in the polymer matrix. The positions of extraneous peaks of MMHFM were not identical to the functionalized MOFs, suggesting the polymer chains intercalation into the clearance of functionalized MOFs particles. Additionally, the characteristic band of amorphous Ultem appeared around $16.1^{\circ}$ that represented 


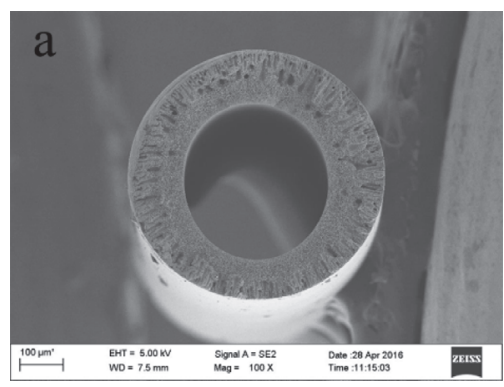

(a)

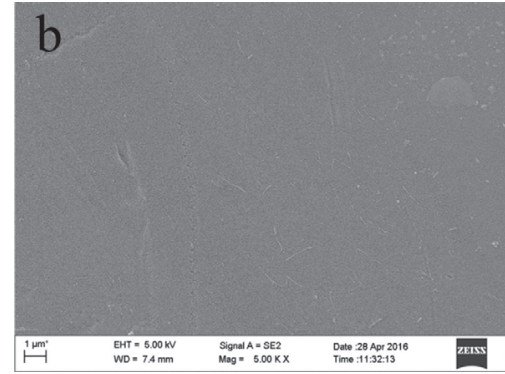

(b)

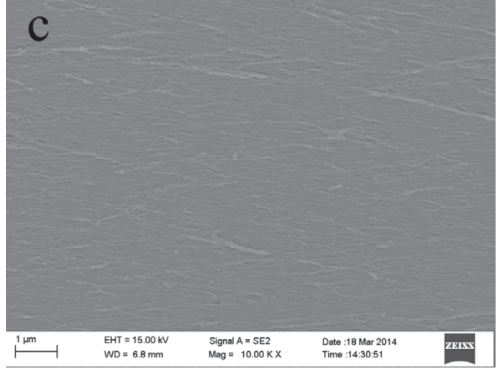

(c)

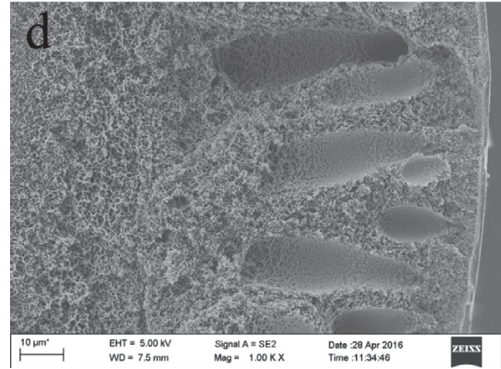

(d)

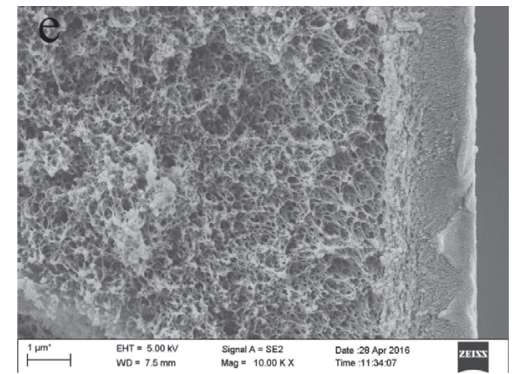

(e)

FIGURE 2: SEM images of cross section (a), outer surface (b), inner surface (c), and skin layer close-up (d, e) of pure Ultem HFM.

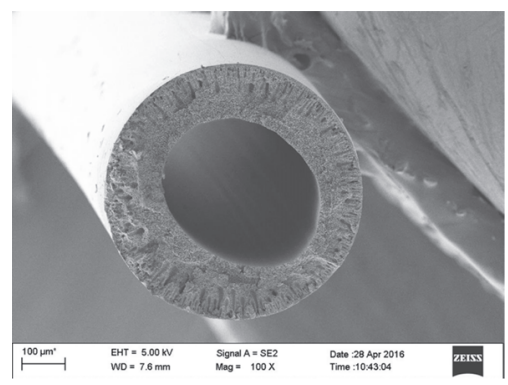

(a)

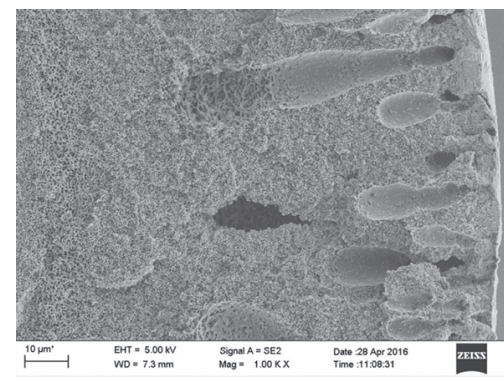

(b)

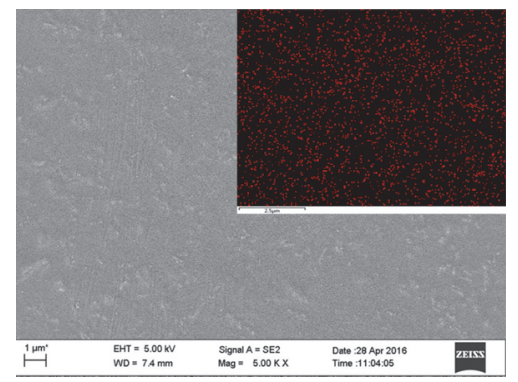

(c)

FIGURE 3: SEM images of cross section (a), skin layer close-up (b), and outer surface (c); the inset is EDX mapping of outer surface (Al: red).

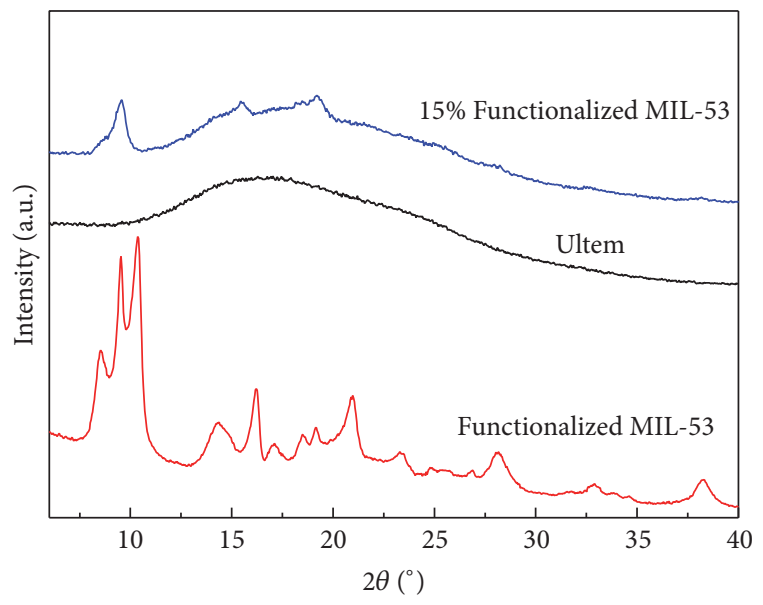

FIGURE 4: X-ray diffractions of functionalized MIL-53, pure Ultem HFM, and Ultem/functionalized MIL-53 MMHFM.
$5.5 \AA$ of the $d$-space value. The band of MMHFM was slightly shifted to lower region which corresponded to a larger $d$-space. This shift revealed the changed arrangement of polymer chains packing with MOFs addition.

As shown in Figure 5, the thermal gravimetric analysis of functionalized MIL-53, pure Ultem HFM, and MMHFM was performed to investigate the membrane thermal stability. A weight loss of functionalized MIL-53 before $140^{\circ} \mathrm{C}$ was caused by the adsorbed water evaporation. Another weight loss stage around $370-515^{\circ} \mathrm{C}$ was ascribed to decomposition of the grafted functional groups. At higher temperature, the organic linkers eliminated from the framework and functionalized MIL-53 transformed to $\mathrm{Al}_{2} \mathrm{O}_{3}$. With addition of functionalized MIL-53 particles, MMHFM achieved a higher decomposition temperature compared with pure Ultem HFM. The decomposition temperature increase for MOFs-containing MMHFM was attributed to higher energy 


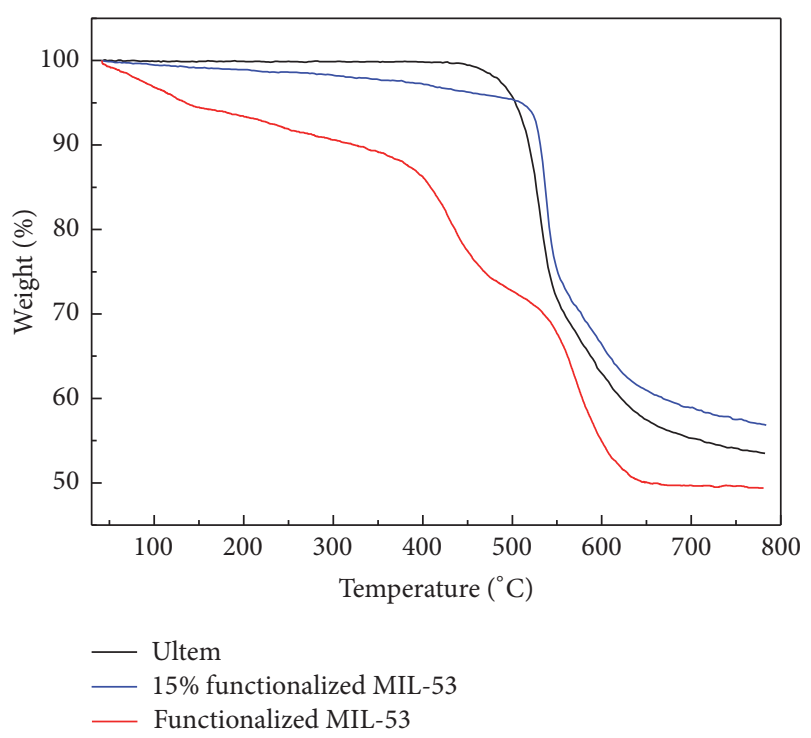

FIGURE 5: TGA curves of functionalized MIL-53, pure Ultem HFM, and Ultem/functionalized MIL-53 MMHFM.

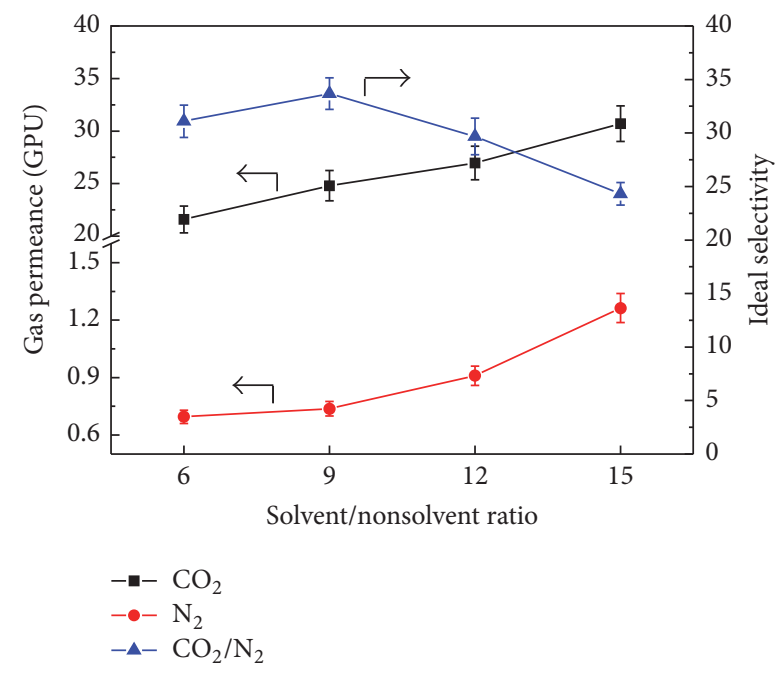

FIGURE 6: Gas permeance and ideal selectivity of MMHFM fabricated from spinning dope with different solvent/nonsolvent ratio.

required for polymer chains movement or segmentation via interaction between polymer matrix and functionalized MIL53. Meanwhile, the shield effect of filler particles would also improve the thermal stability of MMHFM [32].

\subsection{Effect of Solvent/Nonsolvent Ratio in Spinning Dope.} The filler particles significantly affect the phase inversion as indicated by previous references $[31,33]$. The addition of filler would cause the binodal line occurring at lower nonsolvent concentration which reduced the required nonsolvent for separation of dope solution. Figure 6 presents the effect of solvent/nonsolvent ratio in spinning dope on pure gas permeance and ideal selectivity of MMHFM containing the functionalized MIL-53. Gas permeance decreased with the decreased solvent/nonsolvent ratio which was ascribed to the

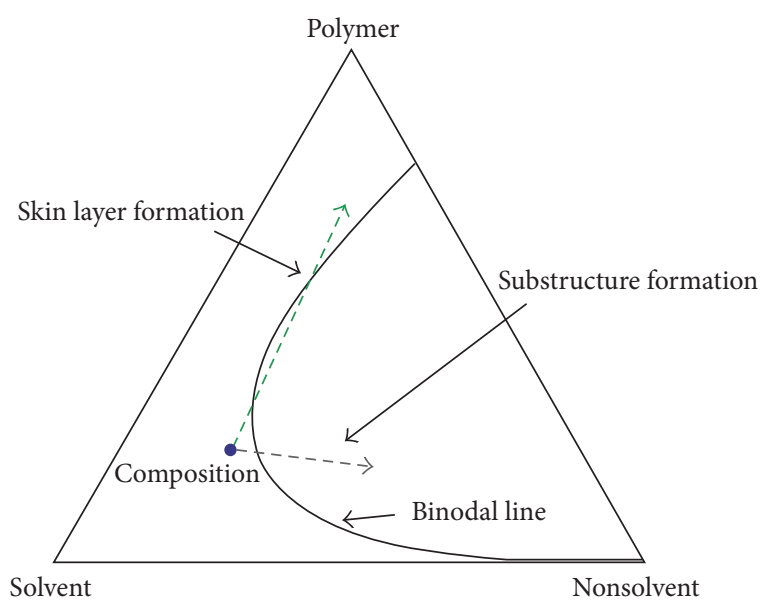

FIgURE 7: Phase diagram demonstrating phase inversion during dry-wet spinning process.

formation of thicker skin layer as Ismail and Lai reported [34]. The evaporation of more volatile nonsolvent could increase polymer concentration of the nascent fiber outer part, resulting in the increment of skin layer thickness. The ideal $\mathrm{CO}_{2} / \mathrm{N}_{2}$ selectivity reached the highest value of 33.7 at the solvent/nonsolvent ratio of 9 . However, excessive nonsolvent in dope caused early phase separation and composition path went across binodal line as indicated by Figure 7 which would trigger defects in the fiber skin in spite of the relatively large thickness of skin layer, thus reducing the ideal selectivity. This was consistent with our previous work [31]. At the solvent/nonsolvent ratio above 9 , on the contrary, the polymer concentration in outermost region of nascent MMHFM was not high enough to form defect-free skin layer. Consequently, the ideal selectivity dropped obviously accompanying with increasing permeance.

\subsection{Effect of Spinning Condition}

3.3.1. Air Gap Distance. Besides the dope composition, the spinning condition was also critical to membrane performance. The outer part cross section of MMHFM prepared through different air gap distances was not identical, especially for the skin layer, as shown in Figure 8 . The MMHFM spun through $2 \mathrm{~cm}$ of air gap distance possessed fine asymmetric structure composed with thin dense skin layer and porous substructure. The functionalized MIL-53 incorporated MMHFM obtained $30.9 \mathrm{GPU}$ of $\mathrm{CO}_{2}$ permeance and also exhibited excellent $\mathrm{CO}_{2} / \mathrm{N}_{2}$ selectivity of 34.7 , as presented in Table 2. In contrast, the MMHFM prepared through a larger air gap distance $(5 \mathrm{~cm})$ had a thicker skin layer (Figure $8(\mathrm{a})$ ), which could be explained by the delayed demixing during phase inversion [34]. The thicker selective layer increased gas transport resistance, which resulted in reduced gas permeance. Additionally, the ideal selectivity also decreased obviously, which should be ascribed to the absorption of moisture through the relatively large air gap. When the air gap distance decreased to $0.8 \mathrm{~cm}$, it was difficult to observe the dense skin layer as shown in Figure 8(c). 
TABLE 2: Effect of spinning condition on gas permeance and ideal selectivity of MMHFM.

\begin{tabular}{lcccc}
\hline Gap distance $(\mathrm{cm})$ & $\mathrm{N}$ Permeance $(\mathrm{GPU})$ & \multicolumn{2}{c}{$\mathrm{N}_{2}$} & $\mathrm{CO}_{2} / \mathrm{N}_{2}$ selectivity \\
\hline 5 & 50 & $\mathrm{CO}_{2}$ & $0.69 \pm 0.03$ & $29.7 \pm 0.7$ \\
2 & 50 & $20.4 \pm 1.0$ & $0.89 \pm 0.03$ & $34.7 \pm 1.0$ \\
0.8 & 50 & $30.9 \pm 1.3$ & $1.61 \pm 0.04$ & $24.8 \pm 1.0$ \\
2 & 10 & $39.8 \pm 1.4$ & $1.01 \pm 0.04$ & $28.0 \pm 1.3$ \\
2 & 90 & $28.3 \pm 1.1$ & $0.74 \pm 0.04$ & $33.6 \pm 1.5$ \\
\hline
\end{tabular}

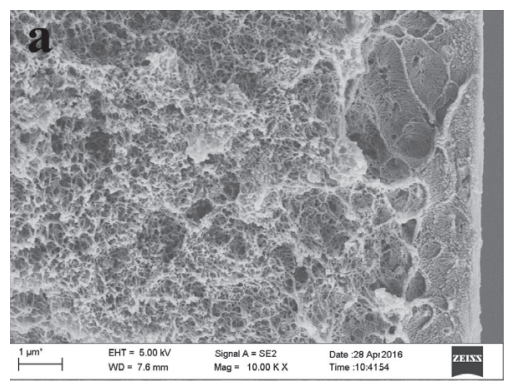

(a)

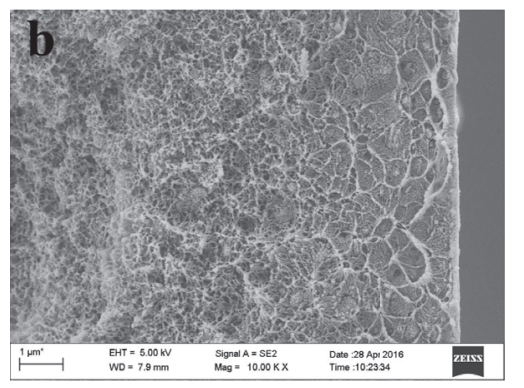

(b)

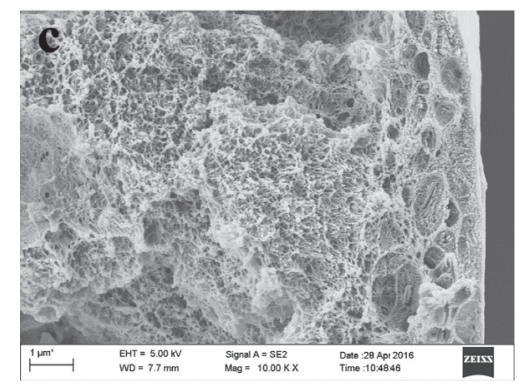

(c)

FIGURE 8: SEM images of outer part cross section of MMHFM prepared through different air gap distances ((a) $5 \mathrm{~cm}$, (b) $2 \mathrm{~cm}$, and (c) $0.8 \mathrm{~cm})$.

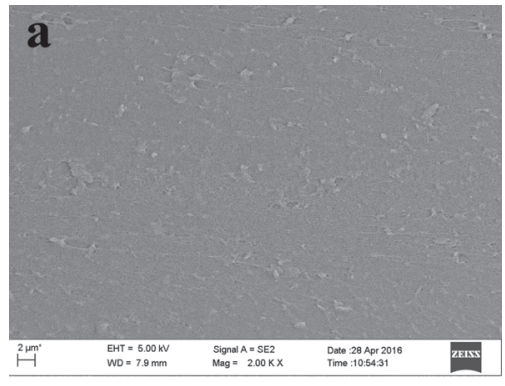

(a)

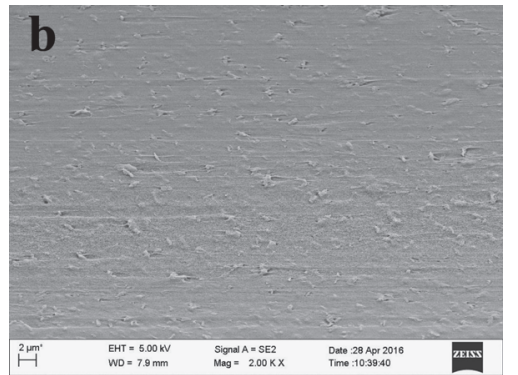

(b)

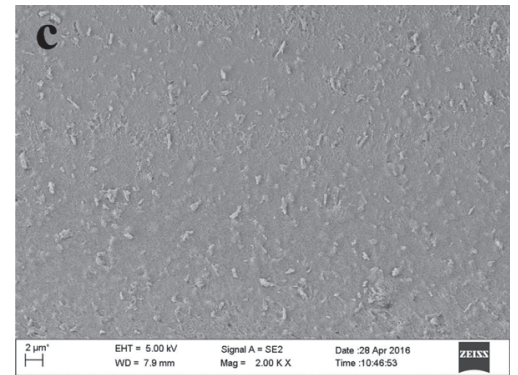

(c)

FIGURE 9: SEM images of inner surface of MMHFMs with different bore fluid compositions ((a) 10\% NMP, (b) 50\% NMP, and (c) $90 \%$ NMP).

Correspondingly, the nonselective pinhole defects would occur in the skin layer inevitably. Therefore, $\mathrm{CO}_{2}$ permeance increased significantly to $39.8 \mathrm{GPU}$, but the ideal $\mathrm{CO}_{2} / \mathrm{N}_{2}$ selectivity was reduced to 24.8 .

3.3.2. Bore Fluid Composition. The bore fluid composition was another important factor that influences the gas separation performance of MMHFM. Three different compositions of bore fluid were investigated in this work. The Ultem/functionalized MIL-53 MMHFM fabricated with the bore fluid containing $10 \mathrm{wt} \%$ NMP showed much lower $\mathrm{CO}_{2} / \mathrm{N}_{2}$ selectivity compared with that of MMHFM with $50 \mathrm{wt} \%$ NMP in the bore fluid. The high water content in the bore fluid would cause early phase separation through the air gap and quickly solidify the inner surface of MMHFM (Figure 9(a)), which would trigger the formation of defective skin layer. When the NMP content increased to $90 \mathrm{wt} \%$, it could be observed that gas permeance decreased to $24.8 \mathrm{GPU}$ for $\mathrm{CO}_{2}$ and $0.74 \mathrm{GPU}$ for $\mathrm{N}_{2}$ (Table 2). The permeance decrease was ascribed to slow coagulation of polymer and formation of dense skin layer. Additionally, the hydrophobic functionalized MIL-53 particles tended to migrate to the inner surface presumably during the transfer between solvent and nonsolvent as indicated by Figure 9(c), which reduced the number of filler particles and fractional free volume in membrane. Therefore, the fast permeation channels decreased substantially, leading to relatively low gas permeance.

\subsection{Comparison of Gas Separation Performance with Pure} Ultem HFM. By varying the dope composition and spinning condition, the optimum permeation performance of MMHFM incorporated with functionalized MIL-53 was achieved. As shown in Table 3, $\mathrm{CO}_{2}$ permeance increased significantly from 12.2 GPU for pure Ultem HFM to 30.9 GPU for MMHFM, which was attributed to the micropores of MOFs that offered extra permeation channels especially in the dense skin layer. The MOFs incorporation would disrupt polymer chain arrangement and thus expand interfacial 
TABLE 3: Gas permeance and ideal selectivity of pure Ultem HFM and MMHFM.

\begin{tabular}{|c|c|c|c|}
\hline \multirow[b]{2}{*}{ Fiber } & \multicolumn{2}{|c|}{ Permeance (GPU) } & \multirow{2}{*}{$\mathrm{CO}_{2} / \mathrm{N}_{2}$ selectivity } \\
\hline & $\mathrm{CO}_{2}$ & $\mathrm{~N}_{2}$ & \\
\hline Pure Ultem HFM & $12.2 \pm 0.4$ & $0.48 \pm 0.02$ & $25.4 \pm 0.8$ \\
\hline MMHFM & $30.9 \pm 1.3$ & $0.89 \pm 0.03$ & $34.7 \pm 1.0$ \\
\hline
\end{tabular}

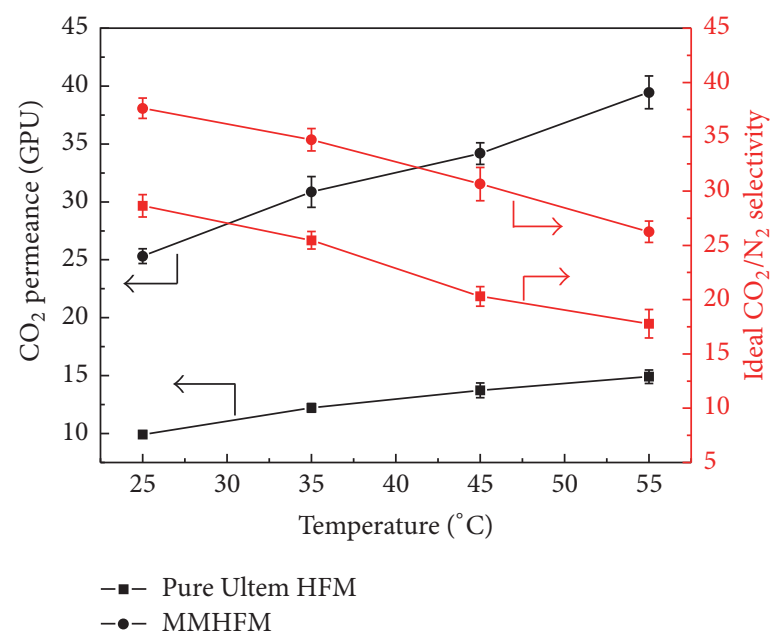

FIGURE 10: Gas permeation properties of pure Ultem HFM and MMHFM plotted as a function of testing temperature.

space, also reducing the permeation resistance. Moreover, the ideal $\mathrm{CO}_{2} / \mathrm{N}_{2}$ selectivity was enhanced to 34.7 by a $37 \%$ increase at the same time. The alkyl chains grafted on MOFs endowed good particle dispersion in polymer matrix and improved polymer/filler interfacial affinity, which avoided the nonselective void formation. Additionally, the aminofunctionalized MIL-53 with $\mathrm{CO}_{2}$ preferential adsorption also increased $\mathrm{CO}_{2}$ solubility $[28,35]$ and then facilitated the improvement of $\mathrm{CO}_{2} / \mathrm{N}_{2}$ selectivity.

3.5. Effect of Testing Temperature on Membrane Performance. The effect of testing temperature on gas permeation properties was also investigated in this work. As shown in Figure 10, the trend of MMHFM was similar to that of pure Ultem HFM following the solution-diffusion based transport mechanism. When the measurement was conducted from $25^{\circ} \mathrm{C}$ to $55^{\circ} \mathrm{C}$, the $\mathrm{CO}_{2}$ permeance of MMHFM increased about $56 \%$ and about $50 \%$ for pure Ultem HFM. The increase in testing temperature not only accelerated the gas diffusion rate but also promoted polymer chain mobility, which lowered the required activation energy for gas permeation and thus increased gas permeance [36]. However, the ideal selectivity decreased with increasing temperature. In order to further investigate the temperature dependency of gas permeance, an Arrhenius type equation was used to represent this activated process, as shown by

$$
P=P_{0} \exp \left(\frac{-E_{p}}{R T}\right),
$$

where $P, P_{0}, E_{p}, R$, and $T$ denote the gas permeation, preexponential factor, activation energy of permeation, universal gas constant, and the absolute temperature, respectively. The activation energy of permeation $\left(E_{p}\right)$ is mainly affected by gas molecular size and solubility $[37,38]$. The noninteracting gas with larger kinetic diameter like $\mathrm{N}_{2}$ has greater $E_{p}$ than the gas with smaller kinetic diameter and better interaction with membrane like $\mathrm{CO}_{2}[38,39]$, so $\mathrm{N}_{2}$ exhibits more temperature dependency of permeance. Hence, gas permeance increased but the selectivity decreased with temperature rising up. Additionally, MMHFM was not damaged by testing from lower temperature up to higher temperature and, returning to lower temperature several times, the gas separation performance was regained.

\section{Conclusion}

MMHFM containing $15 \mathrm{wt} \%$ functionalized MIL-53 was fabricated via the dry-wet phase inversion method. The functionalized MIL-53 particles dispersed homogeneously in the polymer matrix and good interfacial affinity was achieved without nonselective voids or cracks. MMHFM with incorporation of MOFs obtained a higher decomposition temperature compared with pure Ultem HFM because of the difficulty for polymer chain movement and the shield effect of filler particles. MMHFM spun from the dope with appropriate solvent ratio (solvent/nonsolvent ratio was equal to 9) achieved excellent separation performance. Moreover, the spinning condition was also optimized and was better to be chosen $2 \mathrm{~cm}$ of air gap distance and 50\% NMP content in bore fluid composition. By optimizing dope composition, air gap distance, and bore fluid composition, $\mathrm{CO}_{2}$ permeance and ideal $\mathrm{CO}_{2} / \mathrm{N}_{2}$ selectivity of MMHFM reached $30.9 \mathrm{GPU}$ and 34.7, respectively. With respect to the testing temperature, the permeation trend of MMHFM was similar to that of pure Ultem HFM following the solution-diffusion mechanism.

\section{Competing Interests}

The authors declare that there is no conflict of interests regarding the publication of this paper.

\section{Acknowledgments}

This work was supported by the financial support from $\mathrm{Na}$ tional Natural Science Foundation of China (no. 21436009). 


\section{References}

[1] R. W. Baker, "Future directions of membrane gas separation technology," Industrial and Engineering Chemistry Research, vol. 41, no. 6, pp. 1393-1411, 2002.

[2] A. Brunetti, F. Scura, G. Barbieri, and E. Drioli, "Membrane technologies for $\mathrm{CO}_{2}$ separation," Journal of Membrane Science, vol. 359, no. 1-2, pp. 115-125, 2010.

[3] X. Jie, C. Duan, L. Wang et al., "Fabrication of an asymmetric 4,4I-oxydiphthalic anhydride-2,4,6- trimethyl-1,3phenylenediamine/2,6-diaminotoluene copolyimide hollow fiber membrane and its performance for $\mathrm{CO}_{2}$ separation," Industrial and Engineering Chemistry Research, vol. 53, no. 11, pp. 4442-4452, 2014.

[4] L. Xu, C. Zhang, M. Rungta, W. Qiu, J. Liu, and W. J. Koros, "Formation of defect-free 6FDA-DAM asymmetric hollow fiber membranes for gas separations," Journal of Membrane Science, vol. 459, pp. 223-232, 2014.

[5] L. M. Robeson, “The upper bound revisited," Journal of Membrane Science, vol. 320, no. 1-2, pp. 390-400, 2008.

[6] I. F. J. Vankelecom, E. Merckx, M. Luts, and J. B. Uytterhoeven, "Incorporation of zeolites in polyimide membranes," Journal of Physical Chemistry, vol. 99, no. 35, pp. 13187-13192, 1995.

[7] C. M. Zimmerman, A. Singh, and W. J. Koros, “Tailoring mixed matrix composite membranes for gas separations," Journal of Membrane Science, vol. 137, no. 1-2, pp. 145-154, 1997.

[8] H. B. Tanh Jeazet, C. Staudt, and C. Janiak, "Metal-organic frameworks in mixed-matrix membranes for gas separation," Dalton Transactions, vol. 41, no. 46, pp. 14003-14027, 2012.

[9] L. Cao, K. Tao, A. Huang, C. Kong, and L. Chen, "A highly permeable mixed matrix membrane containing CAU-1-NH2 for $\mathrm{H} 2$ and $\mathrm{CO} 2$ separation," Chemical Communications, vol. 49, no. 76, pp. 8513-8515, 2013.

[10] B. Li, H. Wang, and B. Chen, "Microporous metal-organic frameworks for gas separation," Chemistry - An Asian Journal, vol. 9, no. 6, pp. 1474-1498, 2014.

[11] M. J. C. Ordoñez, K. J. Balkus, J. P. Ferraris, and I. H. Musselman, "Molecular sieving realized with ZIF-8/Matrimid ${ }^{\circledR}$ mixed-matrix membranes," Journal of Membrane Science, vol. 361, no. 1-2, pp. 28-37, 2010.

[12] T. Yang, Y. Xiao, and T.-S. Chung, "Poly-/metal-benzimidazole nano-composite membranes for hydrogen purification," Energy and Environmental Science, vol. 4, no. 10, pp. 4171-4180, 2011.

[13] T.-H. Bae and J. R. Long, " $\mathrm{CO}_{2} / \mathrm{N}_{2}$ separations with mixedmatrix membranes containing $\mathrm{Mg}_{2}$ (dobdc) nanocrystals," Energy \& Environmental Science, vol. 6, no. 12, pp. 3565-3569, 2013.

[14] Y. Dai, J. R. Johnson, O. Karvan, D. S. Sholl, and W. J. Koros, "Ultem ${ }^{\circledR} /$ ZIF-8 mixed matrix hollow fiber membranes for $\mathrm{CO}_{2} / \mathrm{N}_{2}$ separations," Journal of Membrane Science, vol. 401402, pp. 76-82, 2012.

[15] H. Hayashi, A. P. Côté, H. Furukawa, M. O’Keeffe, and O. M. Yaghi, "Zeolite A imidazolate frameworks," Nature Materials, vol. 6, no. 7, pp. 501-506, 2007.

[16] R. Banerjee, A. Phan, B. Wang et al., "High-throughput synthesis of zeolitic imidazolate frameworks and application to $\mathrm{CO}_{2}$ capture," Science, vol. 319, no. 5865, pp. 939-943, 2008.

[17] J. Ploegmakers, S. Japip, and K. Nijmeijer, "Mixed matrix membranes containing MOFs for ethylene/ethane separation Part A: membrane preparation and characterization," Journal of Membrane Science, vol. 428, pp. 445-453, 2013.
[18] S. Japip, H. Wang, Y. Xiao, and T. S. Chung, "Highly permeable zeolitic imidazolate framework (ZIF)-71 nano-particles enhanced polyimide membranes for gas separation," Journal of Membrane Science, vol. 467, pp. 162-174, 2014.

[19] S. Japip, Y. Xiao, and T. Chung, "Particle-size effects on gas transport properties of 6FDA-Durene/ZIF-71 mixed matrix membranes," Industrial \& Engineering Chemistry Research, vol. 55, no. 35, pp. 9507-9517, 2016.

[20] S. Couck, J. F. M. Denayer, G. V. Baron, T. Rémy, J. Gascon, and F. Kapteijn, "An amine-functionalized MIL-53 metal-organic framework with large separation power for $\mathrm{CO}_{2}$ and $\mathrm{CH}_{4}$," Journal of the American Chemical Society, vol. 131, no. 18, pp. 6326-6327, 2009.

[21] T. Rodenas, M. Van Dalen, E. García-Pérez et al., "Visualizing MOF mixed matrix membranes at the nanoscale: towards structure-performance relationships in $\mathrm{CO}_{2} / \mathrm{CH}_{4}$ separation over $\mathrm{NH}_{2}$-MIL-53(Al)@PI," Advanced Functional Materials, vol. 24, no. 2, pp. 249-256, 2014.

[22] B. Seoane, C. Téllez, J. Coronas, and C. Staudt, " $\mathrm{NH}_{2}-\mathrm{MIL}$ 53(Al) and $\mathrm{NH}_{2}-\mathrm{MIL}-101(\mathrm{Al})$ in sulfur-containing copolyimide mixed matrix membranes for gas separation," Separation and Purification Technology, vol. 111, pp. 72-81, 2013.

[23] B. P. Biswal, A. Bhaskar, R. Banerjee, and U. K. Kharul, "Selective interfacial synthesis of metal-organic frameworks on a polybenzimidazole hollow fiber membrane for gas separation," Nanoscale, vol. 7, no. 16, pp. 7291-7298, 2015.

[24] A. J. Brown, N. A. Brunelli, K. Eum et al., "Interfacial microfluidic processing of metal-organic framework hollow fiber membranes," Science, vol. 345, no. 6192, pp. 72-75, 2014.

[25] F. Cacho-Bailo, S. Catalán-Aguirre, M. Etxeberría-Benavides et al., "Metal-organic framework membranes on the inner-side of a polymeric hollow fiber by microfluidic synthesis," Journal of Membrane Science, vol. 476, pp. 277-285, 2015.

[26] F. Cacho-Bailo, I. Matito-Martos, J. Perez-Carbajo et al., "On the molecular mechanisms for the $\mathrm{H}_{2} / \mathrm{CO}_{2}$ separation performance of zeolite imidazolate framework two-layered membranes," Chemical Science, vol. 8, no. 1, pp. 325-333, 2017.

[27] T.-S. Chung, L. Y. Jiang, Y. Li, and S. Kulprathipanja, "Mixed matrix membranes (MMMs) comprising organic polymers with dispersed inorganic fillers for gas separation," Progress in Polymer Science, vol. 32, no. 4, pp. 483-507, 2007.

[28] H. Zhu, L. Wang, X. Jie, D. Liu, and Y. Cao, "Improved interfacial affinity and $\mathrm{CO}_{2}$ separation performance of asymmetric mixed matrix membranes by incorporating postmodified MIL53(Al)," ACS Applied Materials \& Interfaces, vol. 8, no. 34, pp. 22696-22704, 2016.

[29] B. Zornoza, A. Martinez-Joaristi, P. Serra-Crespo et al., "Functionalized flexible MOFs as fillers in mixed matrix membranes for highly selective separation of $\mathrm{CO}_{2}$ from $\mathrm{CH}_{4}$ at elevated pressures," Chemical Communications, vol. 47, no. 33, pp. $9522-$ 9524, 2011.

[30] X.-D. Do, V.-T. Hoang, and S. Kaliaguine, "MIL-53(Al) mesostructured metal-organic frameworks," Microporous and Mesoporous Materials, vol. 141, no. 1-3, pp. 135-139, 2011.

[31] H. Zhu, X. Jie, L. Wang, G. Kang, D. Liu, and Y. Cao, "Effect of MIL-53 on phase inversion and gas separation performance of mixed matrix hollow fiber membranes," RSC Advances, vol. 6, no. 73, pp. 69124-69134, 2016.

[32] A. K. Zulhairun, B. C. Ng, A. F. Ismail, R. Surya Murali, and M. S. Abdullah, "Production of mixed matrix hollow fiber membrane for $\mathrm{CO}_{2} / \mathrm{CH}_{4}$ separation," Separation and Purification Technology, vol. 137, pp. 1-12, 2014. 
[33] P. Anadão, L. F. Sato, R. R. Montes, and H. S. De Santis, "Polysulphone/montmorillonite nanocomposite membranes: effect of clay addition and polysulphone molecular weight on the membrane properties," Journal of Membrane Science, vol. 455, pp. 187-199, 2014.

[34] A. F. Ismail and P. Y. Lai, "Effects of phase inversion and rheological factors on formation of defect-free and ultrathinskinned asymmetric polysulfone membranes for gas separation," Separation and Purification Technology, vol. 33, no. 2, pp. 127-143, 2003.

[35] S. Japip, K. Liao, and T. Chung, "Molecularly tuned free volume of vapor cross-linked 6FDA-durene/ZIF-71 MMMs for $\mathrm{H}_{2} / \mathrm{CO}_{2}$ separation at $150^{\circ} \mathrm{C}$," Advanced Materials, vol. 29, no. 4, 2017.

[36] A. L. Khan, S. P. Sree, J. A. Martens, M. T. Raza, and I. F. J. Vankelecom, "Mixed matrix membranes comprising of matrimid and mesoporous COK-12: preparation and gas separation properties," Journal of Membrane Science, vol. 495, pp. 471-478, 2015.

[37] B. D. Freeman, "Basis of permeability/selectivity tradeoff relations in polymeric gas separation membranes," Macromolecules, vol. 32, no. 2, pp. 375-380, 1999.

[38] J. A. de Sales, P. S. O. Patrício, J. C. Machado, G. G. Silva, and D. Windmöller, "Systematic investigation of the effects of temperature and pressure on gas transport through polyurethane/ poly(methylmethacrylate) phase-separated blends," Journal of Membrane Science, vol. 310, no. 1-2, pp. 129-140, 2008.

[39] W.-H. Lin and T.-S. Chung, "Gas permeability, diffusivity, solubility, and aging characteristics of 6FDA-durene polyimide membranes," Journal of Membrane Science, vol. 186, no. 2, pp. 183-193, 2001. 

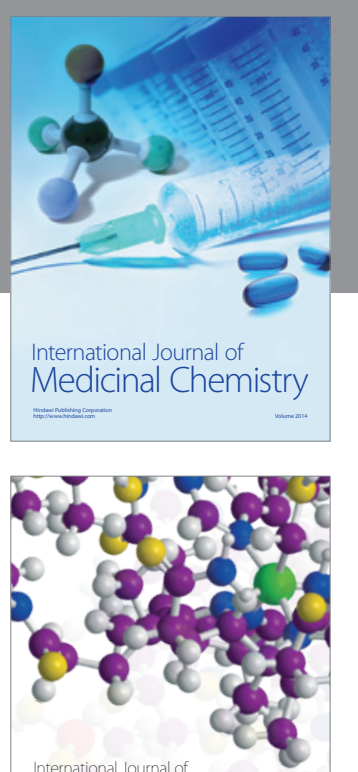

Carbohydrate Chemistry

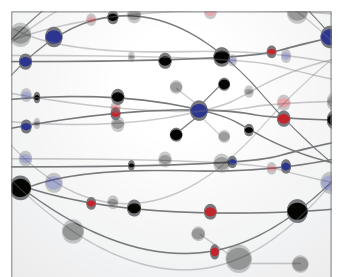

The Scientific World Journal
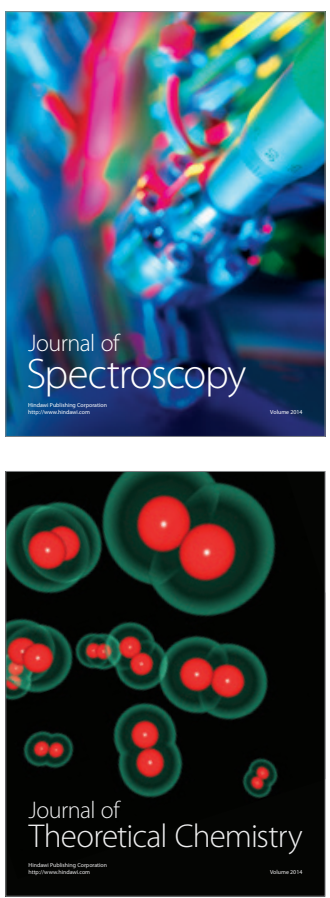
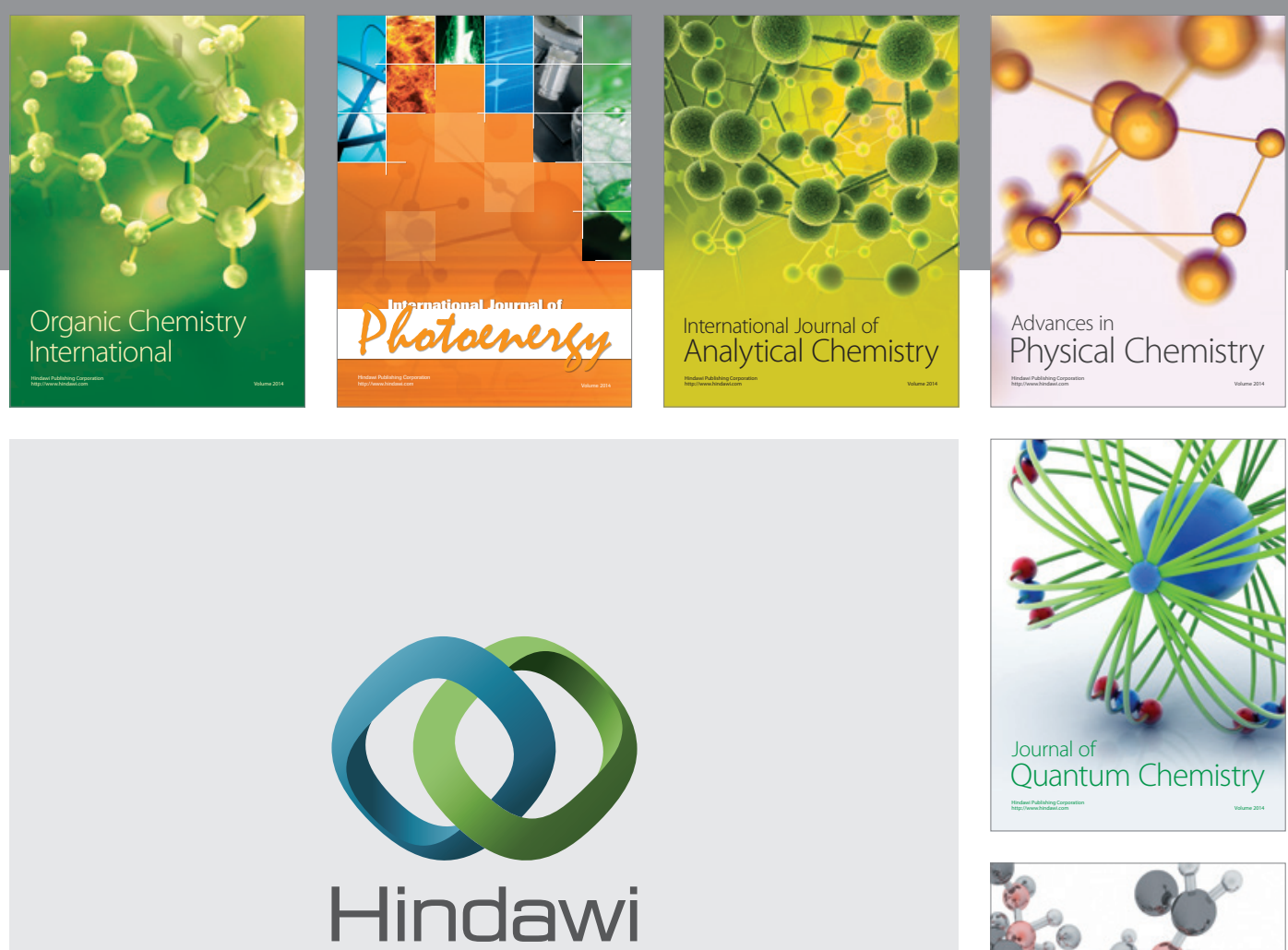

Submit your manuscripts at

https://www.hindawi.com

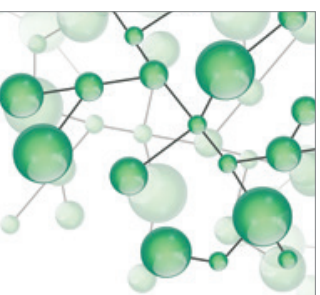

International Journal of

Inorganic Chemistry
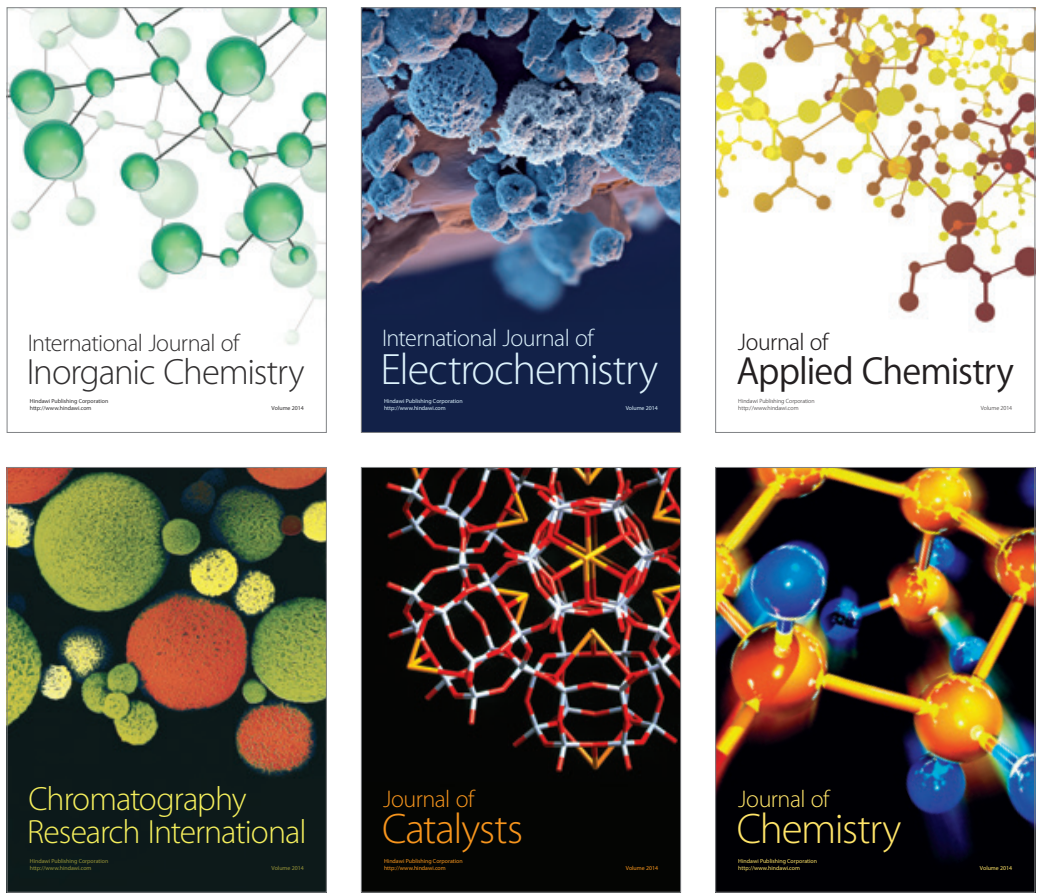

Journal of

Applied Chemistry
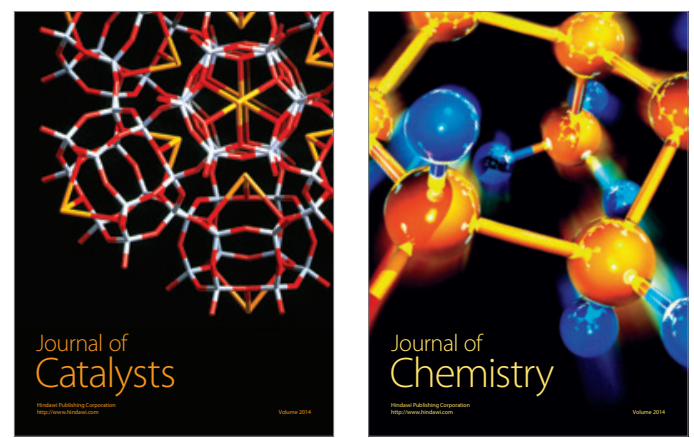
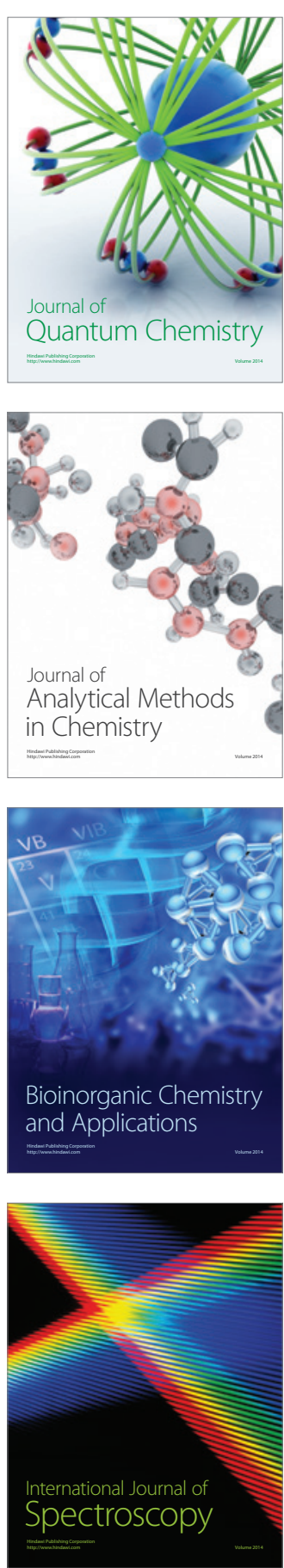